\title{
Phase field approach to spatial perturbations in normal Saffman-Taylor fingers
}

\author{
M. Quevedo-Reyes, ${ }^{1}$ A. Hernández-Machado, ${ }^{2}$ and E. Corvera Poiré ${ }^{1,2, *}$ \\ ${ }^{1}$ Departamento de Física y Química Teórica, Facultad de Química, UNAM. Ciudad Universitaria, México D.F. 04510, Mexico \\ ${ }^{2}$ Departament ECM, Facultat de Física, Universitat de Barcelona, Diagonal 647, E-08028 Barcelona, Spain
}

(Received 6 January 2006; published 23 June 2006)

\begin{abstract}
We make a numerical study of the effect that spatial perturbations have in normal Saffman-Taylor fingers driven at constant pressure gradients. We use a phase field model that allows for spatial variations in the Hele-Shaw cell. We find that, regardless of the specific way in which spatial perturbations are introduced, a lateral instability develops on the sides of the propagating Saffman-Taylor finger. Moreover, the instability exists regardless of the intensity of spatial perturbations in the cell as long as the perturbations are felt by the finger tip. If, as the finger propagates, the spatial perturbations felt by the tip change, the instability is nonperiodic. If, as the finger propagates, the spatial perturbations felt by the tip are persistent, the instability developed is periodic. In the later case, the instability is symmetrical or asymmetrical depending on the intensity of the perturbation.
\end{abstract}

DOI: 10.1103/PhysRevE.73.066308

PACS number(s): 47.54.-r, 05.10.-a, 47.56.+r

\section{INTRODUCTION}

Perturbations to curved fronts out of equilibrium have been widely explored in solidification, where lateral protrusions, called dendrites, grow on the sides of a nearly parabolic front that propagates into an undercooled melt. In the closely related problem of an inviscid fluid displacing a viscous one in a rectangular Hele-Shaw cell, the steady-state shape of the interface is called a Saffman-Taylor finger. Perturbations to the Saffman-Taylor finger have included wires and threads along the flow direction, etching of the plates, and bubbles at the finger tip [1-4]. Theoretically, it has been shown that in the presence of anisotropy [5-9] fingers can be narrower or wider than half of the channel width. Fingers narrower than half of the channel width are called anomalous in order to differentiate them from normal Saffman-Taylor fingers for which the width is larger than half of the channel width. It has been shown [2,3] that anomalous fingers have sides that are unstable to spatial and temporal perturbations. On the other hand, normal Saffman-Taylor fingers have been thought for years as having stable flat sides. Reviews on perturbations to Saffman-Taylor fingers can be found in Refs. $[10,11]$.

Recently, a lateral instability has been reported for fingers propagating in very wide channels [12]. The origin of this instability has not been explained. On the other hand, by means of a numerical integration of a phase field model, a lateral instability of the normal Saffman-Taylor finger was induced dynamically in a controlled manner [13]. The system was driven by a time-dependent pressure gradient, consisting of either periodic forcing or temporal noise, overimposed to the constant pressure gradient needed to drive the interface out of equilibrium. It was numerically demonstrated, that temporal perturbations were able to induce an instability on the normal finger flat sides. Moreover, it was demonstrated that the amplitude of such an instability de-

*To whom correspondence should be addressed. Email address: eugenia.corvera@gmail.com creased with decreasing amplitude of the driving pressure gradient. Experimentally, the effect of quenched disorder on a normal Saffman-Taylor finger has been studied, with the conclusion that quenched noise induces the same type of lateral instability [14] predicted numerically for temporal noise and acts as a selective noise amplifier.

Here we make a numerical study of the effect that spatial perturbations have in normal Saffman-Taylor fingers driven at constant pressure gradients. In particular, we use a phase field model that allows for spatial variations in the HeleShaw cell. We introduce a spatial perturbation consisting of quenched noise distributed all along the cell. We also introduce anisotropy persistent in the direction of flow in two ways, representing, respectively, a parabolic variation of the permeability across the cell and the presence of a stripe along the flow direction. Regardless of the specific way in which spatial perturbations are introduced, we obtain a normal Saffman-Taylor finger with unstable flat sides. For the finger propagating in a cell with quenched noise, we find a lateral instability that is nonperiodic, but, just as the instability of the finger in the presence of temporal noise, it undergoes a process of wavelength selection. For the two cases of perturbations in the form of anisotropy persistent in the flow direction, we find a strictly periodic lateral undulation, with characteristics that agree with the ones found for anomalous fingers, when spatial perturbations are imposed [3].

\section{PHASE FIELD MODEL AND MACROSCOPIC MODEL}

The Saffman-Taylor problem for Newtonian fluids in the infinite-viscosity-contrast limit can be studied by means of a single-order-parameter phase field model $[13,15]$. We take the phase field model of Ref. [15] with a parameter modified to allow for spatial variations in the Hele-Shaw cell.

The mesoscopic equation for the Saffman-Taylor problem with spatial perturbations is

$$
\frac{\partial \phi}{\partial t}=\boldsymbol{\nabla} \cdot[M(\phi, \vec{r}) \boldsymbol{\nabla} \mu(\phi)],
$$

where $\phi$ is an order parameter and $\mu(\phi)$ is a chemical potential that has the explicit form 


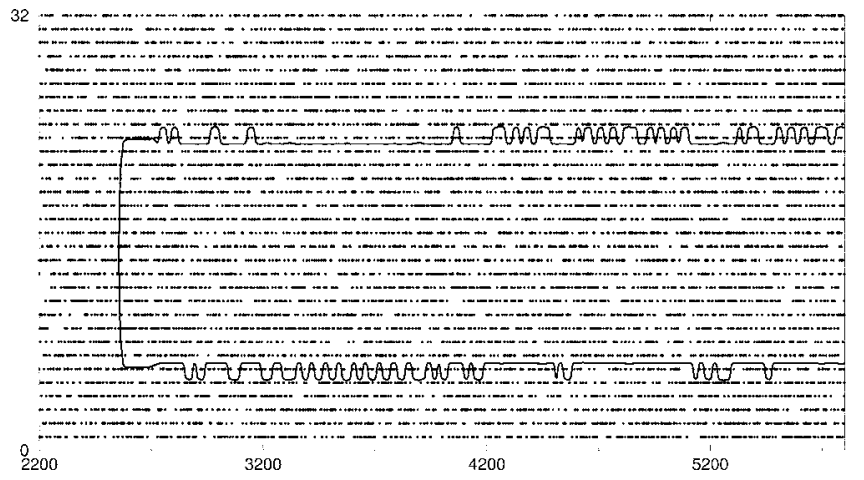

FIG. 1. Finger propagating in a cell with static disorder. $M$ $=1.0$ in $90 \%$ of the cell sites and $M=1.01$ in $10 \%$ of the cell sites. Note that the scale has been compressed in the horizontal direction in order to better appreciate the instability.

$$
\mu(\phi)=-\phi+\phi^{3}-\epsilon^{2} \nabla^{2} \phi
$$

and $\epsilon$ is a parameter proportional to the interface width. The parameter $M(\phi, \vec{r})$ is zero in air $(\phi>0)$ and $M=m[1+\delta(\vec{r})]$ for the viscous fluid $(\phi<0) . m$ is related to the permeability of the viscous fluid in the absence of spatial perturbations, as explained below, and $\delta(\vec{r})$ introduces the possibility of spatial variations. The following boundary condition is imposed at the bulk of the displaced fluid:

$$
\phi\left(x, y \leq y_{\text {tip }}-l\right)=\phi_{B} .
$$

Equation (3) fixes the bulk value $\phi_{B}$ at an arbitrary distance $l$ from the fingertip. $y_{\text {tip }}$ corresponds to the most advanced point of the interface. This creates a ramp that represents the driving force of the system as explained in Ref. [15]. These equations describe the hydrodynamic equations of the macroscopic problem in the sharp interface limit, namely,

$$
\boldsymbol{\nabla} \cdot[1+\delta(\vec{r})] \nabla p=0
$$

which is written from Darcy's law plus the incompressibility of fluids, and the boundary conditions at the fluid-fluid interface, that is, the continuity boundary condition and the local thermodynamic equilibrium condition, that is

$$
\begin{gathered}
v_{n}=-K[1+\delta(\vec{r})] \nabla p \cdot \hat{\mathbf{n}}, \\
\Delta p=-\gamma \kappa .
\end{gathered}
$$

Here $v_{n}$ is the normal velocity of the interface, $\eta$ is the viscosity of the viscous fluid, $b$ is the gap between the cell plates, $\hat{\mathbf{n}}$ is a vector normal to the interface, $\Delta p$ is the pressure drop at the interface, $\gamma$ is the surface tension, and $\kappa$ is the local curvature of the interface. $K[1+\delta(\vec{r})]$ is the space dependent permeability of the viscous fluid.

In order to recover the macroscopic equations from the mesoscopic equations, a matched asymptotic expansion is made and the following identification of parameters has to be done: $p=\phi_{e q} \mu_{1}, K=m / 2 \phi_{e q}^{2}$, and $\gamma=\phi_{e q} \gamma^{\prime} / \Delta \phi$, where $\mu_{1}$ is the first order in $\epsilon$ term of the chemical potential, $\phi_{0}$ is the zero order in $\epsilon$ term of the order parameter, $\Delta \phi=2 \phi_{e q}, \phi_{e q}$ is the bulk value for the order parameter in equilibrium and $\gamma^{\prime}=\int_{-\infty}^{\infty} \partial \phi_{0} / \partial w d w$. The variable $w$ is an inner coordinate of the interface, that is introduced in the expansion, and that is, at any point, perpendicular to it.

It is worth noting that the spatial term of the permeability, $\delta(\vec{r})$, should be small compared to one since we are interested in studying situations in which the single-finger solution exists. We have carried on the numerical integration of Eq. (1) subject to the boundary condition (3). The boundary condition is identical to the one imposed for the steady-state finger, implying that the system is driven at a constant pressure gradient. The initial condition is, in all cases, a slightly perturbed flat interface. Details of the numerical integration are as in Ref. [13].

\section{NATURAL FREQUENCIES}

Before describing the spatial perturbations considered in the present paper, it is convenient to remember that there are two natural frequencies of the steady-state problem. The first characteristic frequency is the finger velocity divided by the finger width, which gives an angular frequency equal to

$$
\omega_{\text {finger }}=2 \pi \frac{U}{\lambda W} .
$$

The other frequency is the one determined by the flow very far from the finger tip, that is, the flow velocity at infinity divided by the channel width, which gives an angular frequency equal to

$$
\omega_{\infty}=2 \pi \frac{V_{\infty}}{W} .
$$

These two frequencies are related to each other since the conservation of matter implies that $U \lambda=V_{\infty}$.

\section{STATIC DISORDER}

We introduce static disorder in the cell by randomly choosing sites where the parameter $M$ takes values that are different than in the rest of the cell. We have arbitrarily set $m=1.0$. The parameter $\delta$ is zero in $90 \%$ of the cell sites and $\delta=0.01$ in $10 \%$ of the cell sites. This is equivalent to say that the permeability of the viscous fluid is different by $1 \%$ in $10 \%$ of the cell sites.

Figure 1 shows a finger propagating in a cell with static disorder. A small-amplitude, long-wavelength, nonperiodic lateral instability develops on the sides of the finger. Black dots indicate places where the parameter $M$ is different than in the rest of the cell.

As the finger propagates into the disorder media a wave is generated at the finger tip and advected far from it towards the sides of the finger. Therefore, the sides of the finger are no longer flat, as in the steady-state case, but develop an instability. The instability undergoes a process of growth, coarsening, and wavelength selection as will be shown. Also, as the wave propagates far from the tip, the amplitude of the instability grows and reaches an almost constant value far from the finger tip. In order to quantify the process of growth, the finger width is measured simultaneously at two different distances from the fingertip $L_{1}$ and $L_{2}$. Due to the 


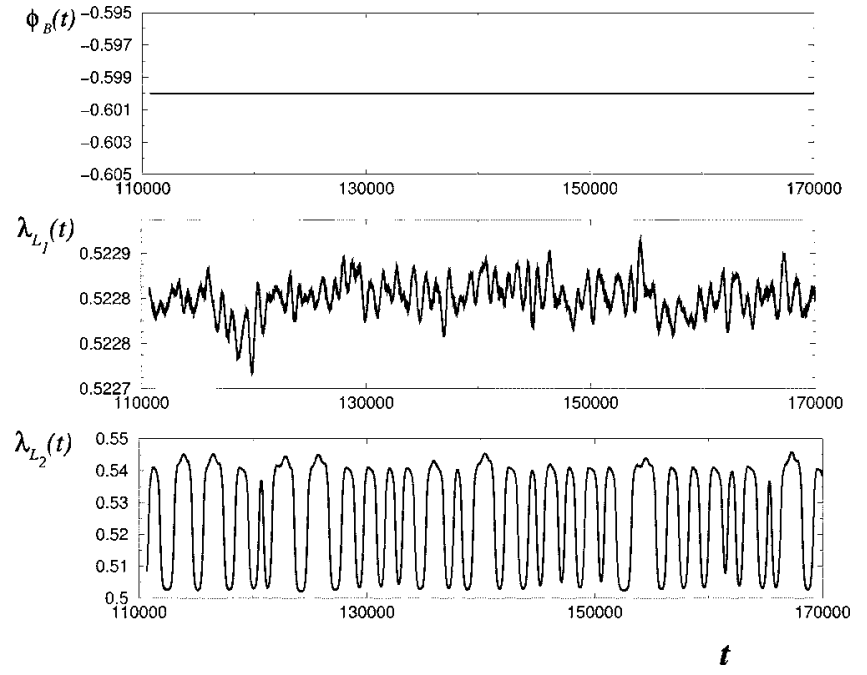

FIG. 2. Bulk order parameter, finger width close to the tip and finger width far from the tip as a function of time for a cell with static disorder. $M=1.01$ in $10 \%$ of the cell sites.

growth process, variations on the finger width measured at distance $L_{1}$, close to the finger tip, have a much smaller amplitude than the ones measured at distance $L_{2}$, far from the finger tip, where the amplitude has reached an almost constant value. Figure 2 illustrates the behavior of the finger width at distances $L_{1}$ and $L_{2}$ as a function of time. The incident signal on the boundary condition of the bulk order parameter has also been plotted in order to emphasize that the system is driven at a constant pressure gradient and that there is no temporal noise associated with the boundary conditions.

From the temporal behavior of the finger width close to the finger tip, we can conclude that the disorder in the cell generates a perturbation close to the finger tip. Comparison of the temporal behavior of the finger width close and far from the finger tip reveals that some modes grow and some modes decay as the perturbation propagates far from the tip. Also, we can see that the amplitude grows as it propagates far from the tip and reaches an almost constant value.

We carry on a frequency distribution analysis, in which we associate a frequency with a distance through the relation

$$
\nu=\frac{U}{\Lambda},
$$

where $U$ is the average finger velocity, and $\Lambda$ is the distance between pairs of adjacent maxima that appear on each side of the finger. This type of analysis was used in Refs. [13,14]. We find a distribution of frequencies with a well-defined peak (see Fig. 3). This indicates that, despite the nonperiodicity of the lateral instability, it undergoes a process of mode selection. The peak has a value close to the characteristic frequency of the flow at infinity. This indicates that the wavelength of the lateral instability is of the order of the channel width. Therefore, the lateral instability described here does not have a dramatic visual effect on the finger, like the instability that causes side branching in solidification, but it causes the finger to have small fluctuations on its width.

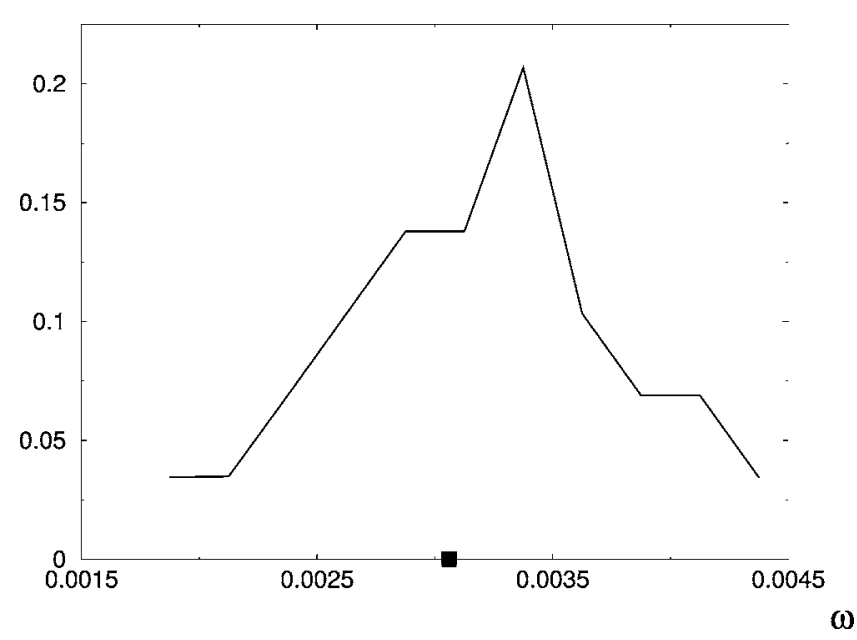

FIG. 3. Frequency distribution for a cell with static disorder. $M=1.01$ in $10 \%$ of the cell sites. The square in the horizontal axis indicates the characteristic frequency at infinity.

\section{Coverage and intensity of static disorder}

There are two variables that may change the properties of the static disorder in the cell: the coverage and the noise intensity. We call coverage, the percentage of sites for which $\delta$ is different from zero. On the other hand, the noise intensity is changed through the value of $\delta$.

The first way in which we study the dependence of the lateral instability with quenched disorder is by keeping constant the value of $\delta$ and varying the coverage. Figure 4 shows profiles for fingers propagating in cells with $1 \%, 10 \%$, and $30 \%$ coverage, respectively. Values of $\delta$ and $m$ have been kept constant and equal to $\delta=0.10$ and $m=1.0$. From the finger profiles, it is clear that the effect of varying the coverage, does not have a strong effect on the instability. It is worth noting that even for extremely small values of coverage the instability exists. That is, even when the permeability is different from the rest of the cell in only $1 \%$ of the cell sites, the flat sides of the finger are unstable to spatial disorder. Frequency distributions for the three coverages can be seen in Fig. 5. It is clear that for all noise intensities, distributions are nonzero on the same range of frequencies and that the peak of the distribution is, in all cases, close to the frequency characteristic of infinity. We can conclude that there is not an obviously quantifiable difference in the distributions and that, even for values of coverages as small as $1 \%$, the instability exists.

The second way in which we study the dependence of the lateral instability with quenched disorder is by keeping constant the coverage and varying the noise intensity through the value of $\delta$. Figure 6 shows profiles for fingers propagating in cells with $10 \%$ coverage and values of $\delta$ equal to $\delta=0.01$, $\delta=0.05$, and $\delta=0.10$, respectively. $m=1.0$ for the three cases. From the finger profiles, it is clear that the intensity of static disorder does not have a strong effect on the instability. Also in this case, it is worth noting that even for extremely small values of $\delta$, the instability exists. That is, even when the permeability is different by only $1 \%$ in $10 \%$ of the cell, the flat sides of the finger are unstable to spatial disorder. Frequency distributions for the three noise intensities can be 


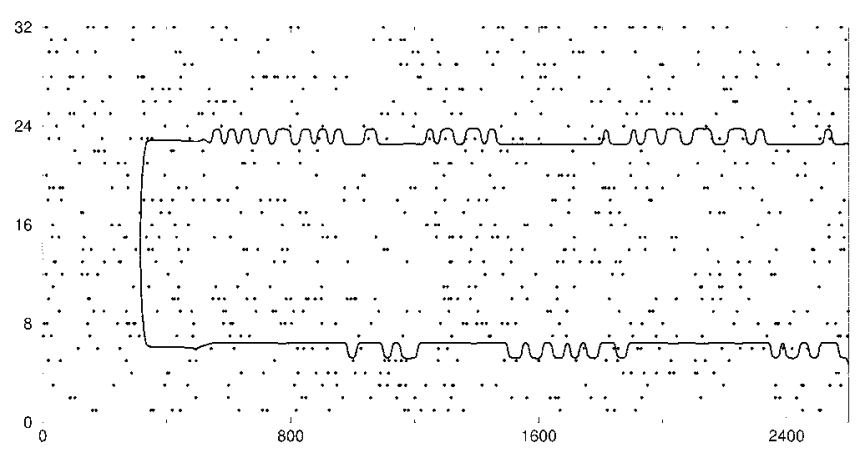

$32\left[\begin{array}{cc}0 . \\ 0\end{array}\right.$

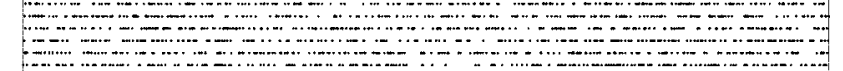

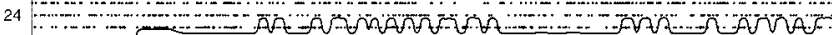
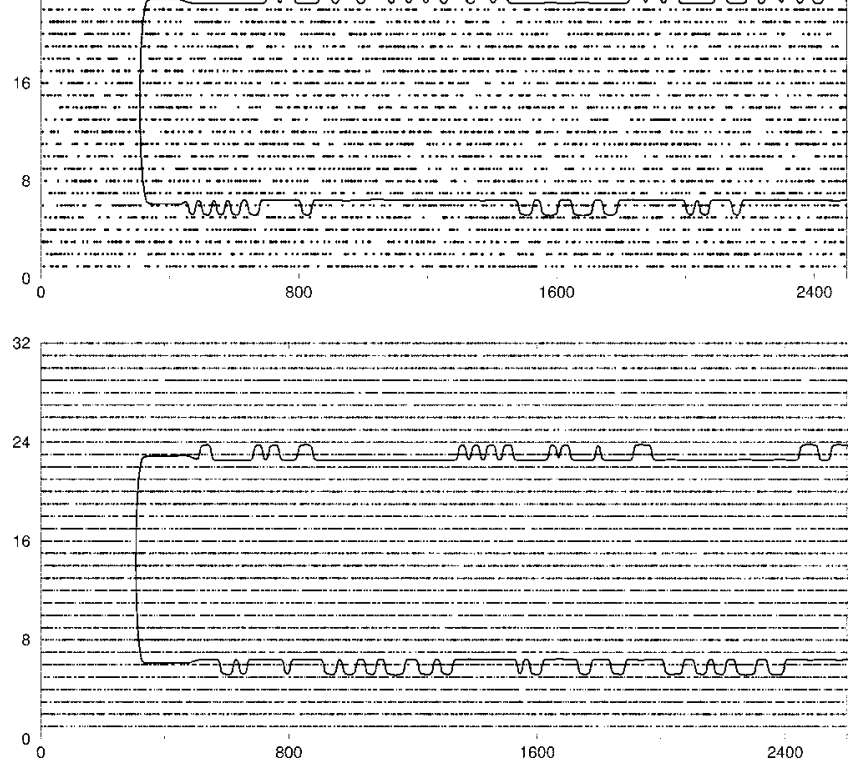

FIG. 4. Finger profiles in cells with $1 \%, 10 \%$, and $30 \%$ coverage, respectively.

seen in Fig. 7. The range of frequencies for which distributions are nonzero seems to be independent of noise intensity. Also, in all cases, the distributions are centered around the frequency characteristic of infinity. We can conclude that the distributions do not depend on the intensity of static disorder and that even for values of $\delta$ as small as $\delta=0.01$ the instability exists.

\section{ANISOTROPY PERSISTENT ALONG THE FLOW DIRECTION}

We introduce two types of perturbations that are persistent along the flow direction. One represents a parabolic variation of the permeability across the cell, the other, the presence of a stripe along the cell. For both situations the permeability of the viscous fluid in front of the finger tip is different than the one on its sides. We find that both ways of introducing anisotropy have similar effects on the Saffman-Taylor finger.

\section{A. Parabolic profile of $M$}

For this perturbation the parameter $M$ is
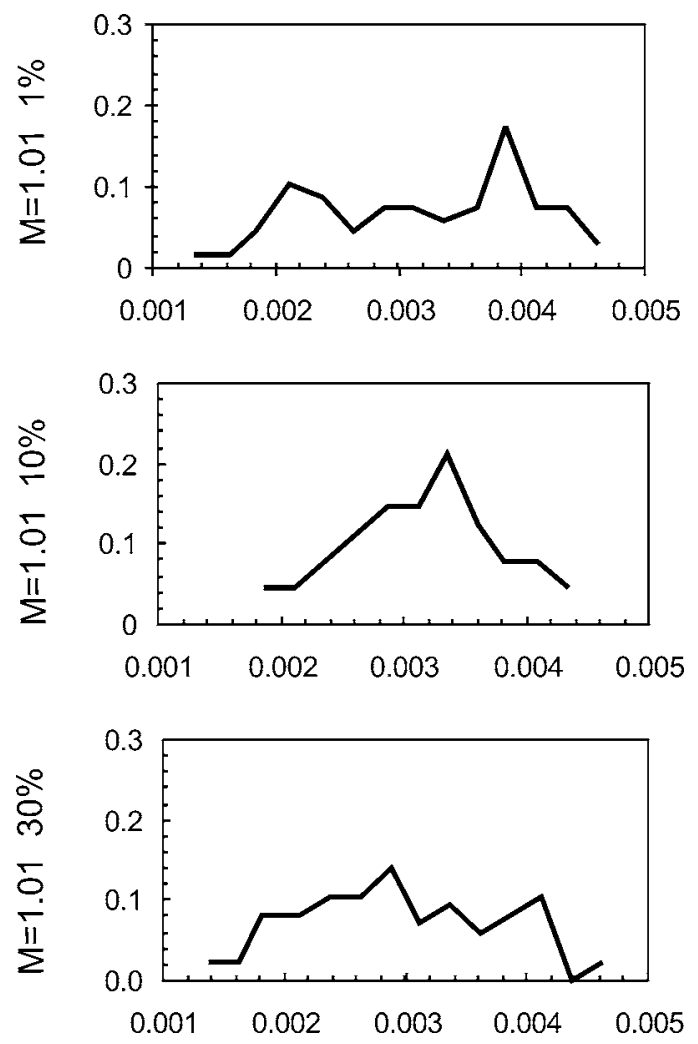

FIG. 5. Frequency distributions for fingers propagating in cells with $1 \%, 10 \%$, and $30 \%$ coverage, respectively.

$$
M= \begin{cases}0 & \text { if } \phi>0 \\ \frac{4\left(M_{\max }-1\right)}{W^{2}}\left(x-\frac{W}{2}\right)^{2}+M_{\max } & \text { if } \phi<0 .\end{cases}
$$

This makes the permeability of the viscous fluid to be $M_{\max }$ in the center of the cell and $M=1$ on the sides of the cell. Since there is no $y$ dependence, the perturbation is persistent along the flow direction $y$. We keep the value of $M_{\max }-1$ relatively small. Since the system is driven at a constant pressure gradient, in the absence of the spatial perturbation $\left(M_{\max }=1\right)$, the dynamics leads to a stable Saffman-Taylor finger with flat sides.

The finger responds by generating a strictly periodic lateral instability close to the finger tip that propagates towards the sides of the finger. Figure 8 shows a typical shape of a finger propagating in a cell with a parabolic profile in the parameter $M$. The scale has been compressed in the flow direction in order to allow for a better appreciation of the periodic nature of the instability.

Our results indicate that in all cases, the lateral instability is strictly periodic but we find that for small perturbations (meaning small values of $M_{\max }-1$ ), the instability is symmetric. That is, the instability is identical at both sides of the finger tip. On the other hand, for large perturbations (meaning large values of $M_{\max }-1$ ), the instability is asymmetric, so as the finger advances the finger body gives the impression of performing a reptating movement, moving now towards the right, then towards the left in alternating turns. 

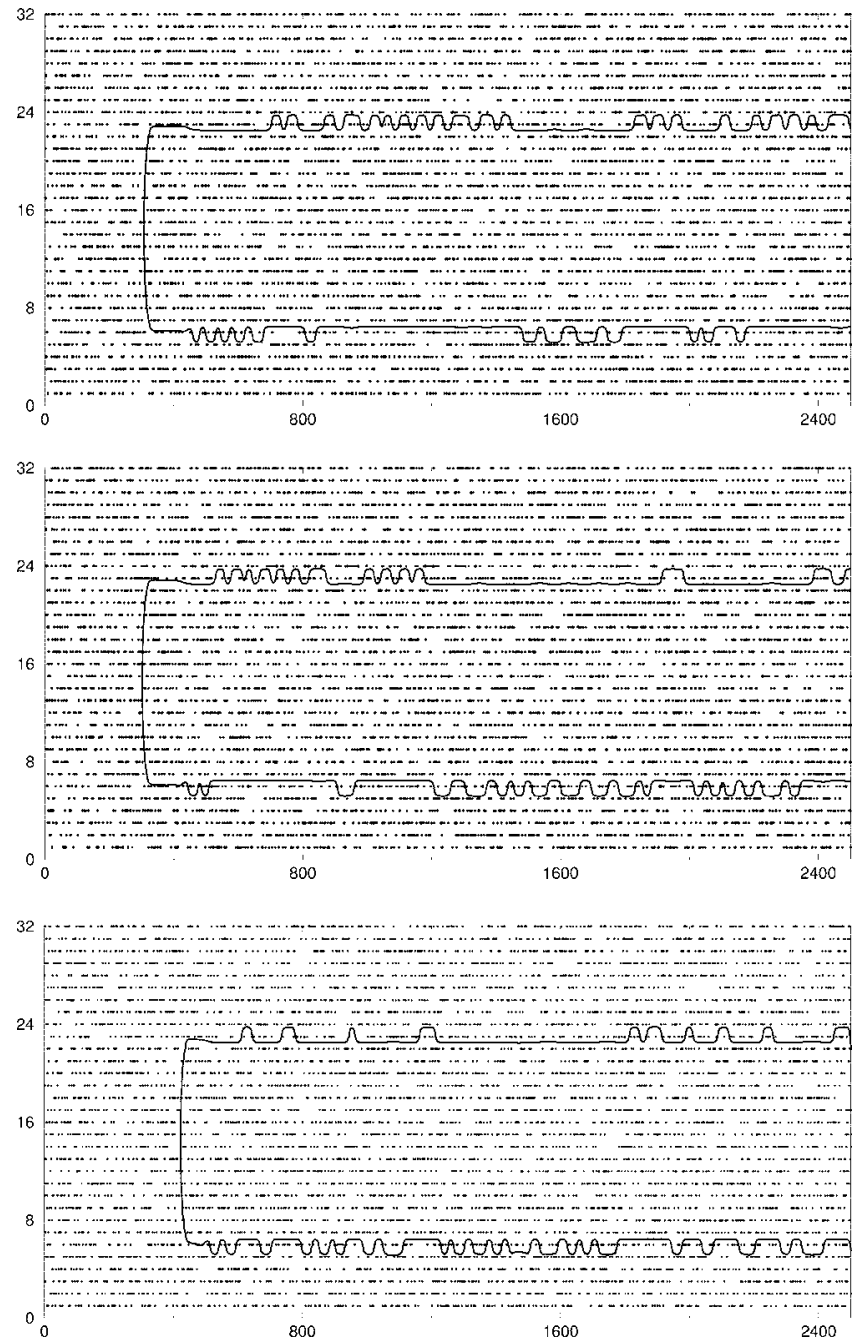

FIG. 6. Fingers propagating in cells with $10 \%$ coverage and different intensities of static disorder: $\delta=0.01, \delta=0.05$, and $\delta$ $=0.10$, respectively.

Our results indicate that for values of $M_{\max }$, corresponding to variations of the permeability of $20 \%$ or less, the instability is symmetric, while for values of $M_{\max }$, corresponding to variations of the permeability between $20 \%$ and $30 \%$, the instability is asymmetric. Finger profiles for different values of $M_{\text {max }}$ are shown in Fig. 9 on a 1:1 scale. For values of $M_{\max }$ corresponding to variations of the permeability in the cell larger than 30\%, we found that the finger develops a nonperiodic lateral instability. For all cases studied, the finger is systematically slightly off center.

Figure 10 illustrates the behavior of the finger width at distances $L_{1}$ and $L_{2}$ as a function of time. $L_{1}$ and $L_{2}$ are defined as in Sec. IV. The incident signal on the boundary condition of the bulk order parameter $\phi_{B}$ has also been plotted in order to emphasize that the system is driven at a constant pressure gradient and that there is no dynamics associated with the boundary conditions. As far as we can observe, the wavelength of the instability does not change as the perturbation propagates far from the tip. In other words, there is no coarsening process, the instability is born with a wavelength that persists as the wave propagates towards the sides
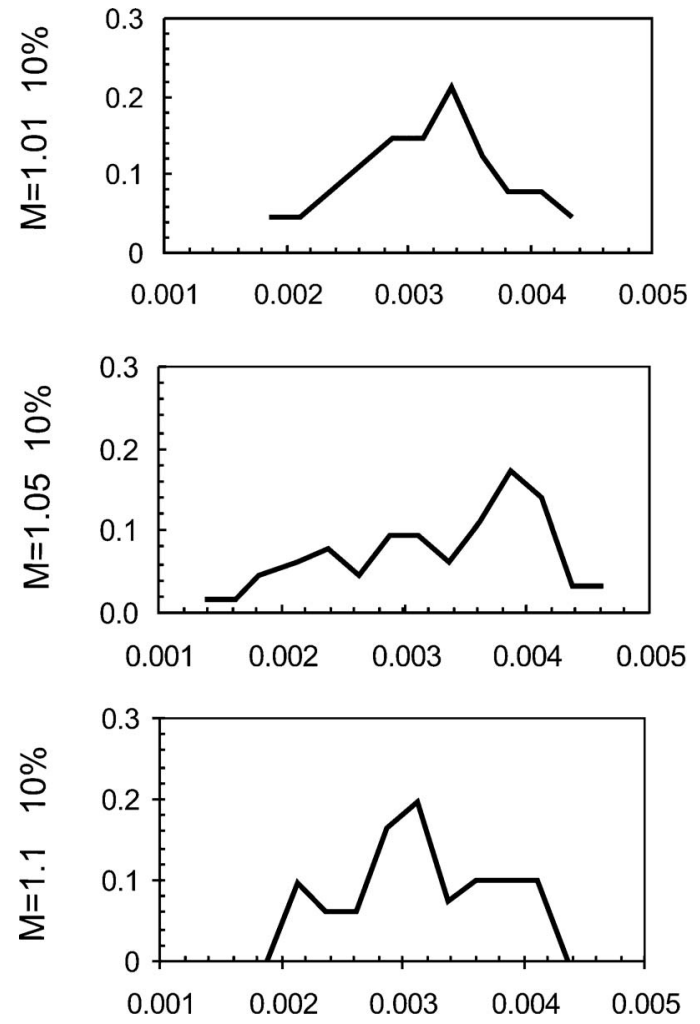

FIG. 7. Frequency distributions for fingers propagating in cells with $10 \%$ coverage and different intensities of static disorder: $\delta$ $=0.01, \delta=0.05$, and $\delta=0.10$, respectively.

of the finger. We found this behavior for all values of $M_{\max }$ studied. On the other hand, the amplitude of the instability, as it is born, is very similar to the amplitude far from the tip, nevertheless, as the instability propagates away from the finger tip, the amplitude grows and its value saturates at a distance of approximately three times the channel width. This would, in principle, allow for an easy experimental observation of the same one.

Our results indicate that the response frequency depends on the value $M_{\max }$ that characterizes the cell and increases monotonically with it regardless of the symmetric or asymmetric nature of the instability. This can be appreciated in Fig. 11. The angular frequency has been computed from the period at a distance $L_{2}$ as $\omega=2 \pi / T$. For all values of $M_{\max }$ studied, the response frequency is between the two characteristic frequencies of the steady state, that for the set of

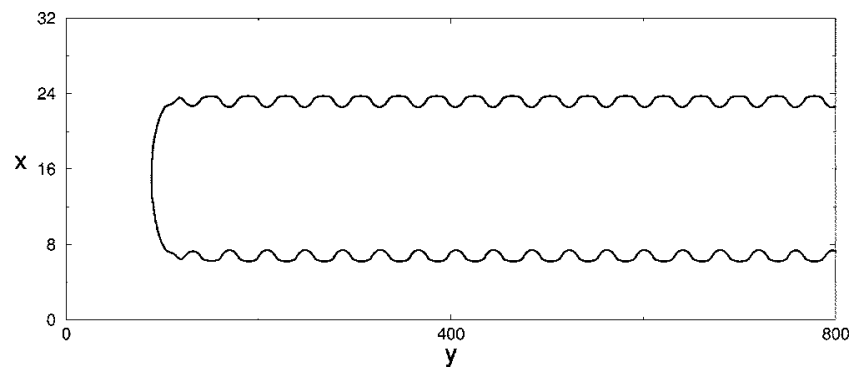

FIG. 8. Finger propagating in a cell with a parabolic profile for $M$. For the figure $M_{\max }=1.1$. 

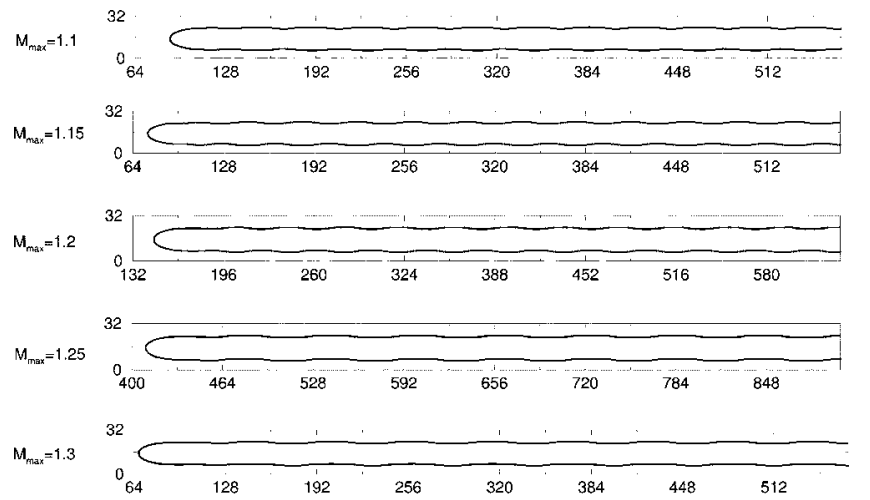

FIG. 9. Finger profiles in a 1:1 scale that show strictly periodic lateral instabilities. Values of $M_{\max }$ are indicated at the left of each profile. The three fingers at the top show symmetric instabilities and the two fingers at the bottom show asymmetric instabilities.

parameters chosen here are $\omega_{\infty}=0.003$ and $\omega_{\text {finger }}=0.011$ in arbitrary dimensions.

\section{B. Stripe along the flow direction}

We introduce a stripe of width $a$ in which the mobility is $M_{t}$ in the following way:

$$
M= \begin{cases}0 & \text { if } \phi>0 \\ M_{t} & \text { if } \phi<0 \text { and } W / 2-a / 2<x<W / 2+a / 2 \\ 1 & \text { if } \phi<0 \text { and } 0<x<W / 2-a / 2 \\ 1 & \text { if } \phi<0 \text { and } W / 2+a / 2<x<W .\end{cases}
$$

The presence of this spatial perturbation induces an instability with the same qualitative characteristics of the one introduced with the parabolic profile of the parameter $M$. That is, a strictly periodic lateral instability is born close to the finger tip and propagates towards the finger sides. For small values of the perturbation, the instability is symmetric and

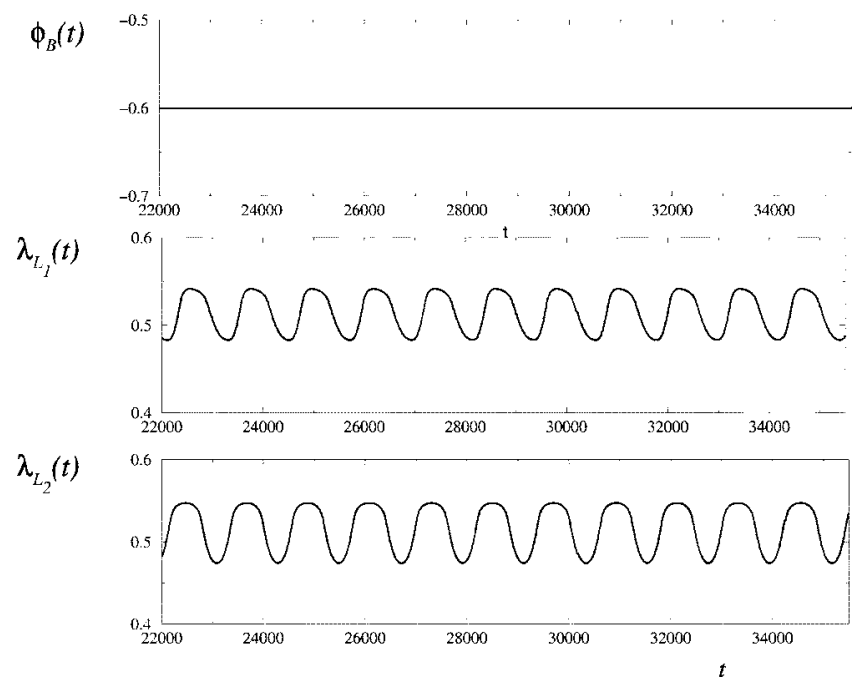

FIG. 10. Bulk order parameter, finger width close to the tip and finger width far from the tip as a function of time for a cell with a parabolic profile for $M . M_{\max }=1.1$ for this figure.

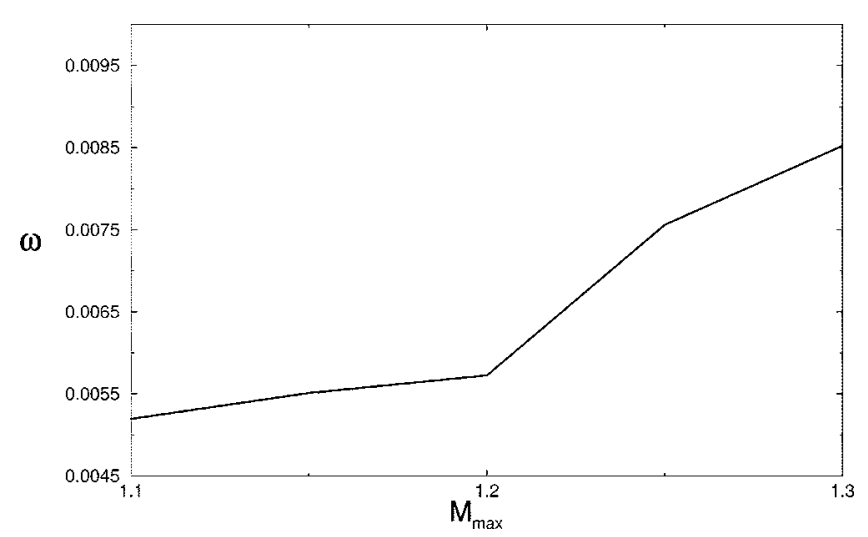

FIG. 11. Response frequency as a function of $M_{\max }$.

for large values of it, the instability is asymmetric. The instability is born with a wavelength that does not change during the propagation and its amplitude saturates at a distance of approximately three times the channel width. Also, just as in the case of the parabolic profile of $M$, we find that the fingers are systematically slightly off center. A profile of a finger propagating in the cell with a stripe of width $a=W / 8$, is shown in Fig. 12.

The overall behavior of the instability is qualitatively like the instability of the finger propagating in a cell with a parabolic profile for $M$. Nevertheless, the quantitative behavior is different. In particular, we find symmetric fingers for values of $M_{t}$ corresponding to variations of the permeability of less than $15 \%$. We find asymmetric fingers for values of $M_{t}$ corresponding to variations of the permeability between $20 \%$ and $25 \%$. For values of $M_{t}$ corresponding to variations in the permeability greater than $25 \%$, we find a nonperiodic instability. Figure 13 shows finger profiles for several values of $M_{t}$. The frequency dependence on the parameter $M_{t}$ is shown in Fig. 14. Also, in this case, for all values of $M_{t}$ studied, the response frequency is between the two characteristic frequencies of the steady state, that for the set of parameters chosen here are $\omega_{\infty}=0.003$ and $\omega_{\text {finger }}=0.011$ in arbitrary dimensions.

For values of the stripe width, $a$, smaller than the one reported, there is no lateral instability. For values larger than $a=W / 8$, we have not been able to find single finger solutions. So, the fact that spatial gradients are large (we have a step in the direction perpendicular to the flow) seems to make critical the width of the stripe.

\section{DISCUSSION}

Static noise induces an instability on the sides of the Saffman-Taylor finger. The instability generates small ampli-

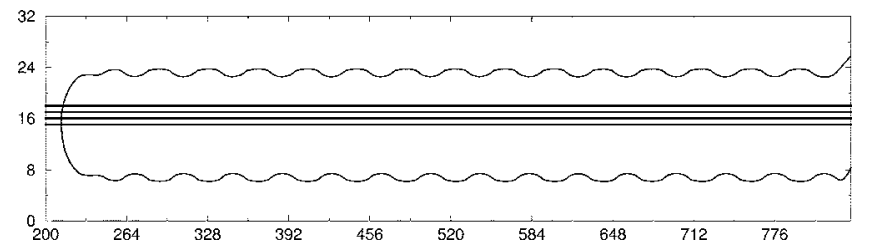

FIG. 12. Finger propagating in a cell with a stripe profile for $M$. For the figure $M_{t}=1.15$. 


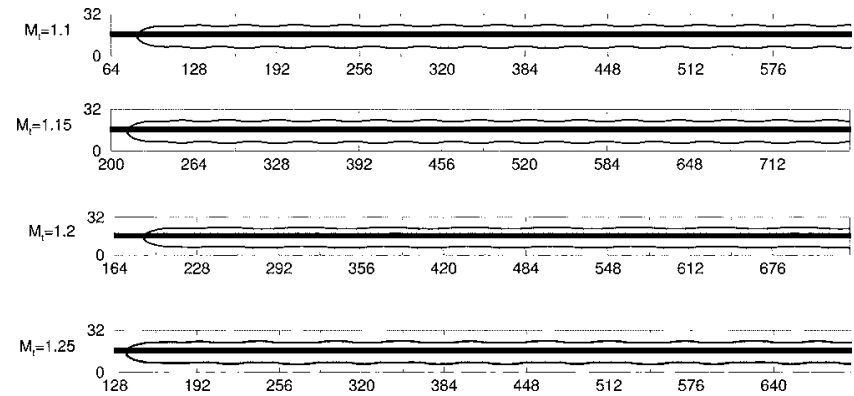

FIG. 13. Finger profiles propagating in a cell with a stripe in a 1:1 scale. Values of $M_{t}$ are indicated at the left of each profile. The two fingers at the top show symmetric instabilities and the two fingers at the bottom show asymmetric instabilities.

tude and long wavelength fluctuations that undergo a process of selection. That is, modes grow and compete dynamically, and the competition gives rise to a nonperiodic structure with a frequency distribution. According to our numerical integration, the distribution is nonzero for a range of frequencies that are around the frequency characteristic of the flow at infinity. The lateral fluctuations have characteristic lengthscales that are of the order of the channel width, which indicates not only that the system is acting as a selective amplifier of static noise, but also that, at a given velocity, the frequency selected is determined by the channel width. The distributions that characterize the instability do not depend strongly on the intensity of static noise. Comparison with numerical studies of the effect that temporal noise has on the Saffman-Taylor finger shape [13] leads to conclude that the effect of temporal noise and static noise are similar since they both generate lateral fluctuations on the finger sides that undergo a process of selection and end up with similar frequency distributions.

In order to explore if other kind of spatial variations leads to the same type of instability, we introduce two different perturbations for which the model parameters varied in the direction transverse to the flow but were constant along the flow. These variations, which represent an anisotropy persistent along the flow direction, induce a strictly periodic instability on the sides of the Saffman-Taylor finger. The instability is characterized by small amplitudes and long-wavelength fluctuations. In these cases, the instability undergoes a process of amplitude growth but not of coarsening, since the wavelength of the instability does not change as the perturbation propagates far from the tip. In other words, the wavelength is selected as the instability is born and persists as the finger propagates into the cell.

The larger the perturbation, that is, the larger the values of $M_{\max }$ or $M_{t}$, the larger the frequency of the instability. Which implies that for large perturbations the wavelengths are small and for small perturbations the wavelengths are large. In all cases, the frequency of the instability is between the two characteristic frequencies of the steady state. This has been observed experimentally for quenched noise [14], and nu-

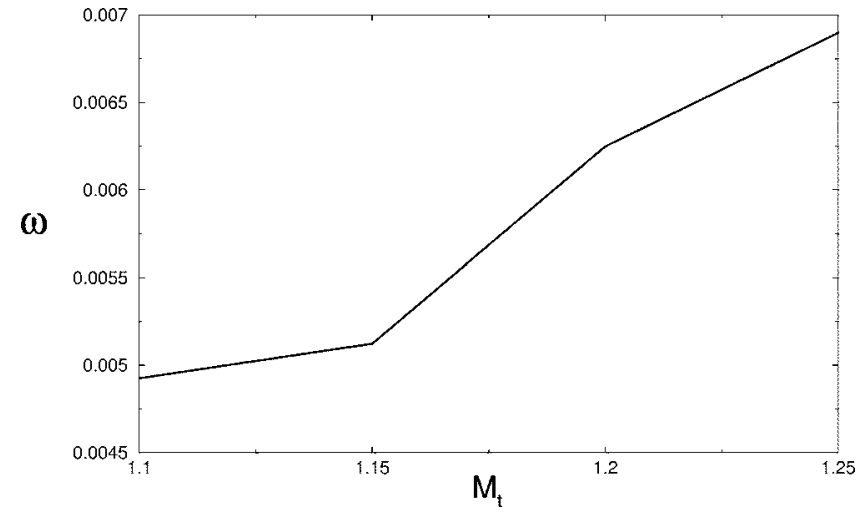

FIG. 14. Response frequency as a function of $M_{t}$.

merically for temporal noise [13]. We find that for small perturbations, meaning small values of $M_{\max }$ or $M_{t}$, the finger develops a symmetric profile and for larger perturbations the finger develops an asymmetric profile. This has been observed before in the context of anomalous fingers [3] where a bubble at the finger tip induces a symmetrical lateral instability when the bubble is small and an asymmetrical lateral instability when the bubble is large. We also observe that our fingers are systematically off center. This has also been observed in the context of anomalous fingers, where a finger propagating into a cell with a thread placed in the center of it, is always off center [2].

Summarizing, we find that, regardless of the specific way in which spatial perturbations are introduced, a lateral instability develops on the sides of the propagating SaffmanTaylor finger. Moreover, as far as we can observe, the instability exists regardless of the intensity of spatial perturbations in the cell as long as the perturbations are felt by the finger tip. If, as the finger propagates, the spatial perturbations felt by the tip change, the instability is nonperiodic. If, as the finger propagates, the spatial perturbations felt by the tip are persistent, the instability developed is periodic. In the later case, the instability is symmetrical or asymmetrical depending on the intensity of the perturbation.

Our studies indicate that the wavelength of the instability is of the order of the cell width, and the saturation distance, from the finger tip, in the case of nonpersistent perturbations, is a few times the cell width. This requires not only longer cells than the ones reported in typical experiments, but requires one to look for the instability, as it was done in Ref. [14], since by nature it is characterized by a low amplitude and a long wavelength.

\section{ACKNOWLEDGMENTS}

The authors thank Rodrigo Ledesma-Aguilar for useful discussions. We acknowledge financial support from the Dirección General de Investigación under Project No. BFM2003-07749-C05-04. E.C.P. acknowledges support from Generalitat de Catalunya and DGAPA, UNAM. 
[1] G. Zocchi, B. E. Shaw, A. Libchaber, and L. P. Kadanoff, Phys. Rev. A 36, 1894 (1987).

[2] M. Rabaud, Y. Couder, and N. Gerard, Phys. Rev. A 37, 935 (1988).

[3] Y. Couder, N. Gérard, and M. Rabaud, Phys. Rev. A 34, 5175 (1986).

[4] K. McCloud and J. V. Maher, Phys. Rev. E 51, 1184 (1995).

[5] A. T. Dorsey and O. Martin, Phys. Rev. A 35, 3989 (1987).

[6] M. Ben Amar, R. Combescot, and Y. Couder, Phys. Rev. Lett. 70, 3047 (1993).

[7] R. Combescot, Phys. Rev. E 49, 4172 (1994).

[8] E. Corvera, H. Guo, and D. Jasnow, Phys. Rev. E 52, 4063 (1995).
[9] D. C. Hong and J. S. Langer, Phys. Rev. A 36, 2325 (1987).

[10] K. McCloud and J. Maher, Phys. Rep. 260, 139 (1995).

[11] Y. Couder, in Perspectives in Fluid Dynamics, edited by G. K. Batchelor, H. K. Moffat, and M. G. Worster (Cambridge University Press, Cambridge, 2000), p. 53.

[12] M. J. Moore, A. Juel, J. M. Burgess, W. D. McCormick, and H. L. Swinney, Phys. Rev. E 65, 030601(R) (2002).

[13] R. Ledesma-Aguilar, M. Quevedo-Reyes, E. Corvera Poiré, and A. Hernández-Machado, Phys. Rev. E 71, 016312 (2005).

[14] M. Torralba, J. Ortín, A. Hernández-Machado, and E. Corvera Poiré, Phys. Rev. E 73, 046302 (2006).

[15] A. Hernández-Machado, A. M. Lacasta, E. Mayoral, and E. Corvera Poiré, Phys. Rev. E 68, 046310 (2003). 